\title{
NCAPG promotes the oncogenesis and progression of non-small cell lung cancer cells through upregulating LGALS1 expression
}

Huanhuan Sun ${ }^{1,2+}$, Hong Zhang ${ }^{3+}$, Yan Yan ${ }^{2+}$, Yushi $\mathrm{Li}^{2}$, Gang Che ${ }^{2}$, Cuiling Zhou ${ }^{2}$, Christophe Nicot ${ }^{4 *}$ and Haiqing $\mathrm{Ma}^{1,2,3^{*}}$

\begin{abstract}
Background: Numerous common oncogenic driver events have been confirmed in non-small cell lung cancer (NSCLC). Although targeted therapy has revolutionized NSCLC treatment, some patients still do not respond. NCAPG, also known as non-SMC condensin I complex subunit G, was positively associated with proliferation and migration in several tumor types.
\end{abstract}

Methods: We used transcriptional sequencing and TCGA database analysis to identify NCAPG as a new therapeutic target for NSCLC. The oncogenic roles of NCAPG in NSCLC tumor growth and metastasis were detected in vitro and in vivo. Ncapg ${ }^{+/+}$or $\mathrm{Ncapg}^{+/-}$mice with urethane treatment were analyzed for oncogenesis of NSCLC.

Results: We investigated NCAPG as a new oncogenic driver which promoted NSCLC tumorigenesis and progression. We used transcriptome sequencing and the Cancer Genome Atlas (TCGA) database analysis to screen and found that NCAPG was negatively correlated with NSCLC survival. Using immunohistochemistry, we demonstrated that NCAPG overexpression was an independent risk factor for NSCLC survival. Functionally, NCAPG knockdown inhibited proliferation, migration, and invasion of NSCLC cells in vitro and in vivo. We exposed wildtype or $\mathrm{Ncapg}^{+/-}$mice to urethane and discovered that urethane-induced lung tumors were reduced in $\mathrm{Ncapg}^{+/-}$mice. Mechanistically, the function of NCAPG in promoting initiation and progression of NSCLC was closely related to LGALS1, which was also upregulated in NSCLC and might interact directly with NCAPG.

Conclusions: This study indicates that NCAPG is one of the essential factors for NSCLC oncogenesis and progression, providing a new target for prognosis prediction and treatment of NSCLC.

Keywords: NSCLC, NCAPG, LGALS1, Oncogenesis, Progression

*Correspondence: cnicot@kumc.edu; mahaiqing@gdph.org.cn †Huanhuan Sun, Hong Zhang and Yan Yan contributed equally to this work.

${ }^{1}$ Medical Research Center, Guangdong Provincial People's Hospital, Guangdong Academy of Medical Sciences, 106 Zhongshan Er Rd, Guangzhou 510080, Guangdong, China

${ }^{4}$ Department of Pathology and Laboratory Medicine, University of Kansas Medical Center, 3901 Rainbow Boulevard, Kansas City, KS 66160, USA

Full list of author information is available at the end of the article

\section{Introduction}

The discovery of actionable oncogenic drivers includes epidermal growth factor receptor (EGFR) [1], anaplastic lymphoma kinase (ALK) [2], mesenchymal-epithelial transition factor (MET) [3], Kirsten rat sarcoma 2 viral oncogene homolog (KRAS) [4], serine/threonine-protein kinase b-raf (BRAF) [5], and ROS1 proto-oncogene receptor tyrosine kinase (ROS1) [6], which have revolutionized the molecular targeted therapy of NSCLC. However, these specially targeted inhibitors only original author(s) and the source, provide a link to the Creative Commons licence, and indicate if changes were made. The images or other third party material in this article are included in the article's Creative Commons licence, unless indicated otherwise in a credit line to the material. If material is not included in the article's Creative Commons licence and your intended use is not permitted by statutory regulation or exceeds the permitted use, you will need to obtain permission directly from the copyright holder. To view a copy of this licence, visit http://creativecommons.org/licenses/by/4.0/. The Creative Commons Public Domain Dedication waiver (http://creativeco mmons.org/publicdomain/zero/1.0/) applies to the data made available in this article, unless otherwise stated in a credit line to the data. 
provide clinical benefits to patients with related driver gene mutations, and these patients still have a challenge with acquired drug resistance. Discovering new potential biomarkers is urgently needed for NSCLC treatment.

To identify new therapeutic targets for NSCLC, we used transcriptional sequencing and TCGA database analysis and found that NCAPG might be involved in NSCLC development regulation. The non-SMC subunits are responsible for ATP-dependent DNA supercoiling and chromosome segregation [7]. NCAPG, also known as non-SMC condensin I complex subunit G, was first isolated from HeLa cell nuclei and demonstrated to regulate the location of DNA on chromosomes [8]. Oncogenic NCAPG is positively associated with $\mathrm{S}$ phase cell cycle arrest, proliferation and migration in several tumor types, including gastric cancer [9], breast cancer [10], hepatocellular carcinoma (HCC) [11], and etc. Meanwhile, the clinical importance of NCAPG's biology is that its overexpression was associated with poor prognosis in HCC [12] and renal cell carcinoma [13]. However, the role of NCAPG in NSCLC and its related mechanisms require elucidation.

Here, we demonstrated the ability of NCAPG to promote the proliferation, migration, invasion, and metastasis in NSCLC. By analyzing the Cancer Genome Atlas (TCGA) database and clinical samples of NSCLC, we found that NCAPG was the only differentially expressed gene that negatively correlated with the survival of NSCLC patients. Additionally, we investigated the function of NCAPG on tumorigenesis of NSCLC by knocking it out in a murine lung cancer model. Furthermore, LGALS1 was identified to be interacted with NCAPG to participate in NSCLC progression. As a result, we hypothesized that NCAPG could be a potential biomarker and therapeutic target in NSCLC.

\section{Materials and methods}

\section{TCGA and GEO database analyses}

Between 1991 and 2013, data on 999 adenocarcinoma and squamous cell carcinoma NSCLC cases were extracted from the TCGA database (https://cancergeno me.nih.gov/). These data were used to analyze differential expression, correlation, and patient survival. Table S1 contains clinical data on the 999 cases. The data of GSE102287 were obtained from Gene Expression
Omnibus (GEO) database (https://www.ncbi.nlm.nih. gov/gds/). These data were analyzed by differential expression analysis.

\section{Cell lines and cultures}

NSCLC cell lines including HCC827, H460, H1299, H1975, A549, and PC9 were purchased from the American Type Culture Collection (ATCC). H460, H1299, H1975, and HCC827 were cultured in ATCC-formulated RPMI-1640 medium. PC9 cells were cultured in highglucose Dulbecco's modified eagle medium (DMEM). A549 cells were cultured in an ATCC formulated F-12 K medium. Each medium contained $10 \%$ FBS and $100 \mathrm{U} /$ $\mathrm{mL}$ penicillin. These cells were cultured at $37^{\circ} \mathrm{C}$ and in a humidified incubator at 5\% CO2.

\section{NSCLC specimens}

Between February 2010 and November 2014, 156 pairs of NSCLC tumor and adjacent normal tissue specimens were obtained from patients who underwent surgeries at the Fifth Affiliated Hospital of Sun Yat-sen University and Sun Yat-sen University Cancer Center. Table 2 lists the clinicopathological characteristics of 156 patients. None of these patients received radiotherapy or chemotherapy before surgery, and all received standard therapy following surgery. The histologic types were determined following the criteria set by the World Health Organization (WHO). All procedures followed were in accordance with the ethical standards of the responsible committee on human experimentation (institutional and national) and the Helsinki Declaration of 1975, as revised in 2000. All patients provided informed consent to participate in the study.

\section{Proximity ligation assays (PLA)}

PLA was carried out with Duolink Detection Kit (Sigma, DUO92101-1KTT) according to manufacturer's instructions. Briefly, fixed and permeabilized cells were incubated for $30 \mathrm{~min}$ at $37^{\circ} \mathrm{C}$ in a blocking solution. The cells were incubated with primary antibodies diluted in Duolink antibody diluents overnight at $4^{\circ} \mathrm{C}$. After washes, the cells were incubated for $60 \mathrm{~min}$ at $37^{\circ} \mathrm{C}$ with appropriate PLA probes. Following washes, circularization and ligation of appropriate oligonucleotides were

\footnotetext{
(See figure on next page.)

Fig. 1 Identification of NCAPG expression in NSCLC. A Gene sequencing volcano plots of NSCLC tumor tissues and adjacent normal tissues (blue indicates downregulated genes; red indicates upregulated genes). B GO-BP analysis of upregulated genes in tumor tissues. C Heatmaps of all 39 upregulated genes involved in cell cycle, mitotic division, cell division, and mitotic cell cycle. D The 13 upregulated genes highly expressed in NSCLC tumor tissues from the TCGA database. E The relationship between NCAPG expression and survival in NSCLC from the TCGA database. F, G NCAPG expression level in the TCGA and GEO databases. (*** $p<0.001)$
} 


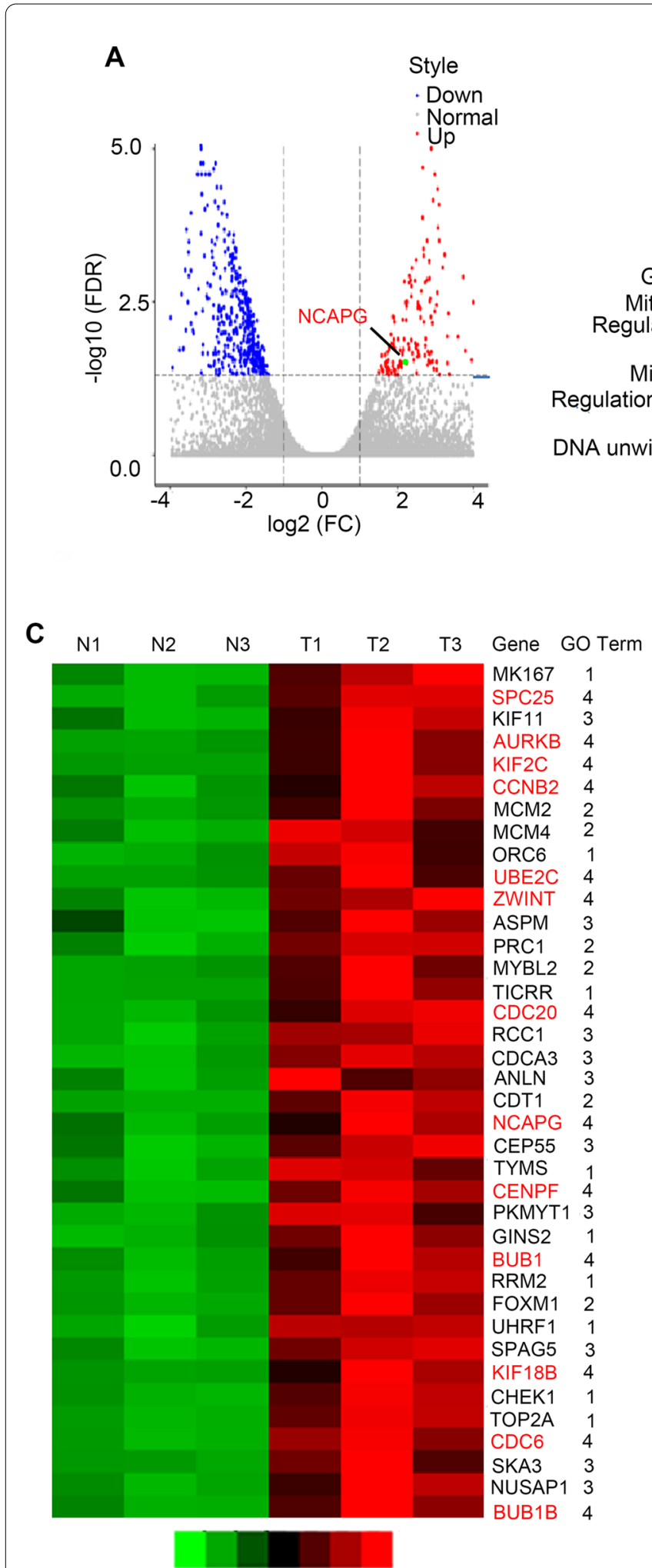

B

GO-Analysis_BP

$-\log _{10}(P$-value $)$

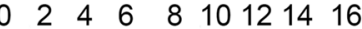

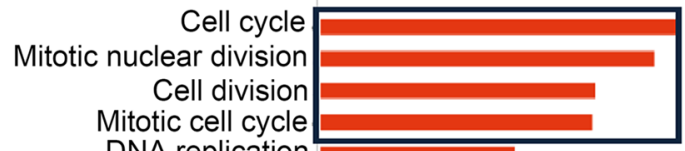

Chromosome segregation

G1/S transition of mitotic cell cycle

Mitotic spindle assembly checkpoint

Regulation of chromosome segregation

Cell proliferation

Mitotic sister chromatid segregation

Mitotic cytokinesis

volved in DNA replication

Mitotic spindle organization

D
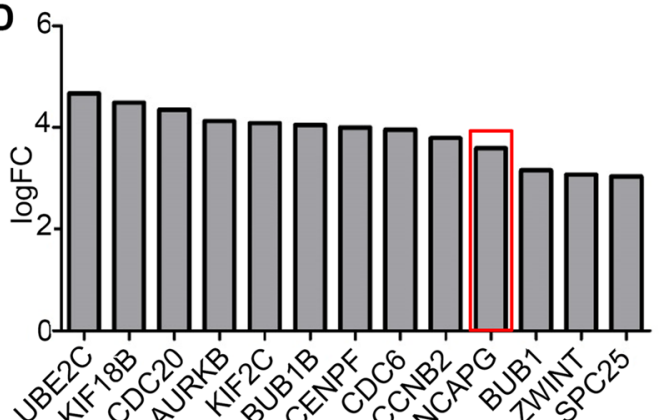

E

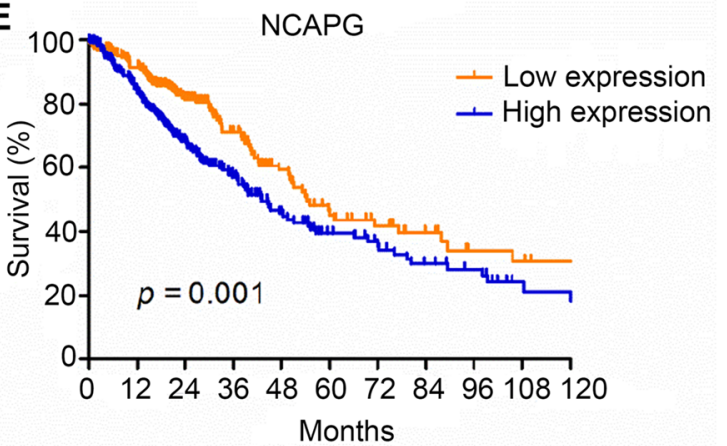

F
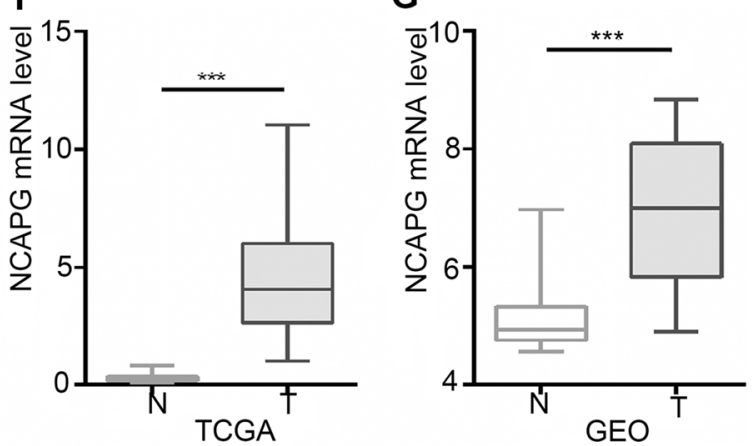

Fig. 1 (See legend on previous page.) 
performed in ligase-containing solution for $30 \mathrm{~min}$ at $37^{\circ} \mathrm{C}$. The cells were then rinsed briefly and incubated for $100 \mathrm{~min}$ at $37^{\circ} \mathrm{C}$ with an amplification solution. After PBS washes, coverslips were mounted with Duolink in Situ Mounting Medium with DAPI. Samples were analyzed using a laser-scanning confocal microscope and multispectral imaging flow cytometry. These experiments were repeated at least three times.

\section{5-ethynyl-2'-deoxyuridine assay}

A549 and H1299 cells were transfected with NCAPG shRNA and/or LGALS1 overexpression vectors. And the cells were cultured in medium contained 5-ethynyl-2'deoxyuridine (EdU) $(30 \mu \mathrm{M})$ (Cell Light EdU DNA imaging Kit, Guangzhou RiboBio, China) for $2 \mathrm{~h}$. The levels of EdU were detected by flow cytometry according to the manufacturer's protocol.

\section{Animal experiments}

All animals were purchased from Sun Yat-sen University Animal Center. All animal experiments were approved by the Ethics Committees of Guangdong Provincial People's Hospital and the Fifth Affiliated Hospital of Sun Yat-sen University. This study was performed following the ethical standards of the Declaration of Helsinki. Additionally, all institutional and national guidelines for the care and use of laboratory animals were followed. To evaluate the role of NCAPG in the growth of NSCLC, $20 \mathrm{BALB} / \mathrm{c}$ nude female mice (8weeks old) were randomly divided into two groups ( $n=10$ per group). A549 $\left(5 \times 10^{6}\right)$ and $\mathrm{H} 1299\left(2 \times 10^{6}\right)$ cells transfected with either LV-shNCAPG or LV-shCtrl were injected subcutaneously into each axilla of mice separately. Tumor volumes

Table 1 NSCLC stage and their correlation with NCAPG expression in TCGA database

\begin{tabular}{lllll}
\hline Characteristic & \multicolumn{2}{l}{ NCAPG expression } & $p$-value & $\mathbf{r}$ \\
\cline { 2 - 3 } & Negative $\mathbf{n}$ (\%) & Positive $\mathbf{n}(\%)$ & & \\
\hline Tumor stage & & & & \\
PT1 & $175(63.6)$ & $100(36.4)$ & $<0.001$ & 0.17 \\
pT2 & $249(45.9)$ & $294(54.1)$ & & \\
pT3 & $44(39.3)$ & $68(60.7)$ & & \\
pT4 & $17(42.5)$ & $23(57.5)$ & & \\
Node metastasis & & & & \\
pN0 & $343(54.2)$ & $290(45.8)$ & $<0.001$ & 0.127 \\
pN1/ pN2/pN3 & $142(42.1)$ & $195(57.9)$ & & \\
TNM stage & & & & \\
I & $290(58.0)$ & $210(42.0)$ & $<0.001$ & 0.198 \\
II & $115(41.4)$ & $163(58.6)$ & & \\
III & $67(40.9)$ & $97(59.1)$ & & \\
IV & $13(46.4)$ & $15(53.6)$ & & \\
\hline
\end{tabular}

were measured every four days and calculated with the formula: volume $=0.5 \times$ length $\times$ width $^{2}$. The mice were sacrificed after $4-5$ weeks, and the tumors were weighed.

For the in vivo pulmonary metastasis murine model, ten NOD/SCID mice (6weeks old) were injected through the tail vein with $\mathrm{H} 1299\left(1 \times 10^{6}\right)$ cells transfected with either LV-shNCAPG or LV-shCtrl. The mice were sacrificed after 31 days and the lungs were harvested and analyzed for the presence of metastatic tumors.

Heterozygous Ncapg mice were generated by Cyagen Biosciences Inc. (Suzhou, China) in order to intercross for homozygotes. Exons 2, 3, 4 and 5 of mouse Ncapg were knockout by the CRISPR Cas9 system. The following mouse Ncapg sgRNA sequences were used: sgRNA F: GTGTGTCGAGTGTAGCGGCGGGG; sgRNA R: GAG AGGGTGGGGCATTATACTGG. Genetic deletion analysis for Ncapg was examined by multiplex genomic DNA PCR according to the manufacturer's protocol (Cyagen Biosciences Inc). Primer sequences were as follows: F1: 5'-AGTGACTTGTTGATCTGCGCAG-3'; R1: 5'-GCC AAACTCCTACATCACCTATG-3'; R2: 5'-CTCTCA ACGCCTTTGGATTATCG-3'. However, no homozygous Ncapg knockout mice have ever been born. A urethane-induced lung tumor model was used to investigate the role of Ncapg in NSCLC initiation. A total of $20 \mathrm{Ncapg}^{+/-}$mice and $20 \mathrm{Ncapg}^{+/+}$C57BL/6 mice were intraperitoneally injected with urethane $(1.0 \mathrm{mg} / \mathrm{g}$ body weight) once per week for six weeks. The mice were sacrificed 12 weeks after urethane treatment, and the lungs were harvested and further analyzed. Genotype results of mouse embryonic fibroblasts (MEFs) from offspring mice embryos are provided in Table $\mathrm{S} 2$.

\section{Immunoprecipitation-MS}

Previous studies demonstrated the use of immunoprecipitation-MS to detect NCAPG- interacting proteins $[14,15]$.

\section{Statistical analysis}

A paired-samples $t$-test was used to compare mRNA and protein expression of NCAPG and LGALS1 in NSCLC tumors and the paired adjacent normal tissue samples. The $\chi^{2}$ test was used to analyze the relationship between NCAPG expression and various clinicopathologic characteristics. The Kaplan-Meier method was used to calculate the survival curves, and the log-rank test was used to compare them. For univariate and multivariate analyses, Cox proportional hazard regression model was used to determine the effect of clinicopathologic variables and NCAPG expression on survival. All statistical analyses were conducted using SPSS 16.0 software (SPSS, Chicago, IL), and a $p$-value $<0.05$ was considered statistically significant [16]. In addition, the remaining methods 


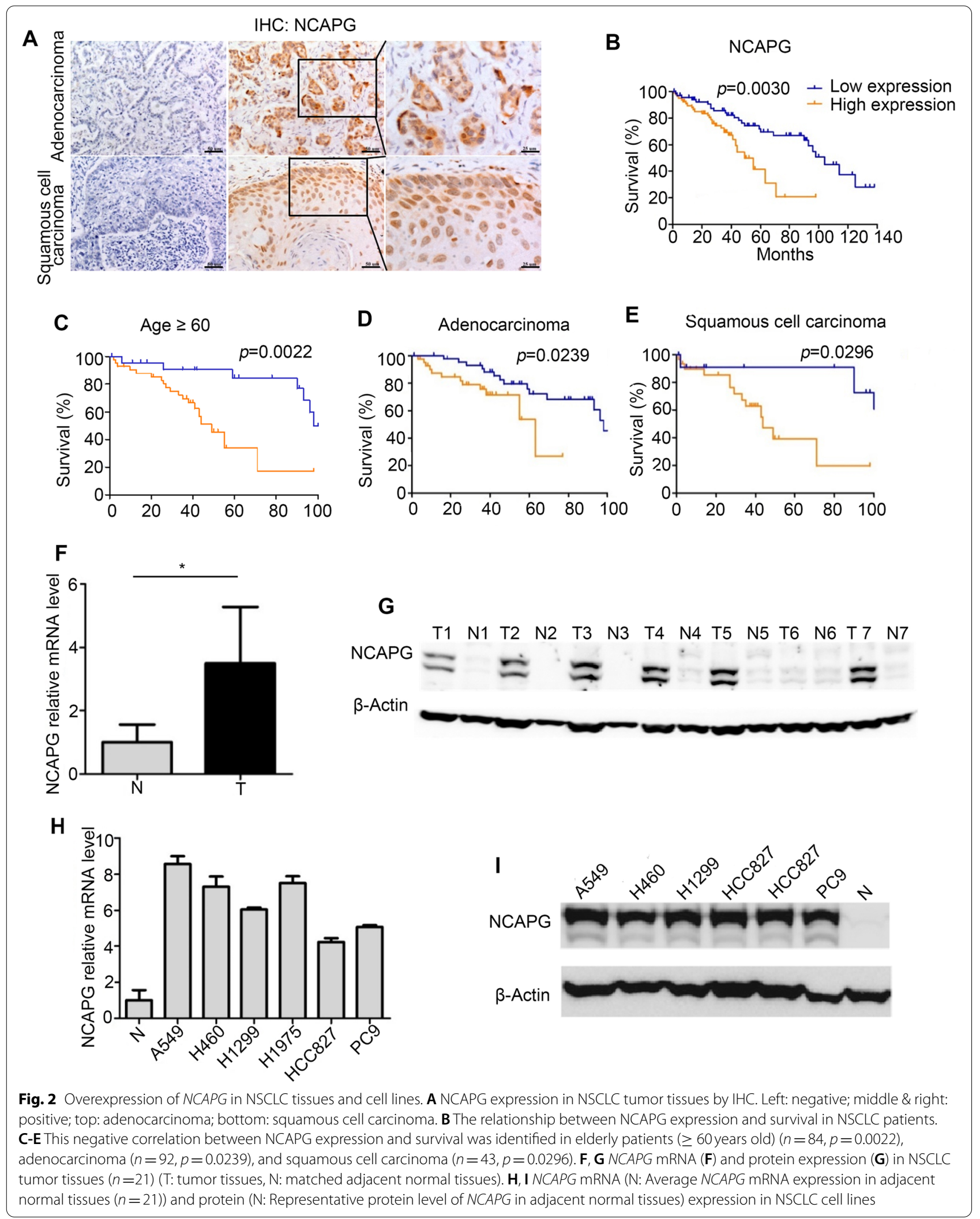


Table 2 Patients' characteristics and their association with NCAPG expression

\begin{tabular}{|c|c|c|c|}
\hline $\begin{array}{l}\text { Characteristic } \\
\text { NCAPG expression }\end{array}$ & $\begin{array}{l}\text { Negative }(N=72), \\
n(\%)\end{array}$ & $\begin{array}{l}\text { Positive }(N=84), \\
\text { n (\%) }\end{array}$ & $P$-value \\
\hline Gender & & & 0.014 \\
\hline Male & $34(38)$ & $56(62)$ & \\
\hline Female & $38(58)$ & $28(42)$ & \\
\hline Age & & & 0.020 \\
\hline$<60$ & $46(55)$ & $38(45)$ & \\
\hline$\geq 60$ & $26(36)$ & $46(64)$ & \\
\hline Tumor stage & & & 0.523 \\
\hline pT1 & $12(39)$ & $19(61)$ & \\
\hline pT2 & $38(53)$ & $34(47)$ & \\
\hline pT3 & $14(56)$ & $11(44)$ & \\
\hline pT4 & $2(50)$ & $2(50)$ & \\
\hline Node metastasis & & & 0.235 \\
\hline pNO & $47(54)$ & $40(46)$ & \\
\hline $\mathrm{pN} 1 / \mathrm{pN} 2$ & $18(43)$ & $24(57)$ & \\
\hline TNM stage & & & 0.537 \\
\hline | / / & $48(48)$ & $52(52)$ & \\
\hline III / IV & $24(43)$ & $32(57)$ & \\
\hline Histological type & & & 0.006 \\
\hline Adenocarcinoma & $49(53)$ & $43(47)$ & \\
\hline Squamous cell carcinoma & $12(28)$ & $31(72)$ & \\
\hline Differentiated degree & & & 0.881 \\
\hline Poorly differentiated & $13(43)$ & $17(57)$ & \\
\hline Moderately differentiated & $43(43)$ & $58(57)$ & \\
\hline Highly differentiated & $5(50)$ & $5(50)$ & \\
\hline
\end{tabular}

are presented in Supplementary Methods, Figures and Tables.

\section{Results}

\section{NCAPG is upregulated in NSCLC}

To identify new therapeutic targets of NSCLC, we performed transcriptome sequencing on three assembled paired tumor tissue samples and adjacent normal tissues. We identified 415 downregulated genes and 158 upregulated genes (Fig. 1A, $\log 2 \mathrm{FC}>1$ or $<-1$, false discovery rate $(\mathrm{FDR})<0.05)$. GO analysis revealed that 39 upregulated genes were frequently involved in four biological processes including cell cycle, mitotic nuclear division, cell division, and mitotic cell cycle (Fig. 1B, C). Surprisingly, 13 genes were simultaneously involved in these four biological processes (Fig. 1C). Moreover, TCGA data analysis showed that the 13 genes were overexpressed in NSCLC tumor tissues (Fig. 1D, $\log 2 \mathrm{FC}>2, p<0.001$ ), but only NCAPG was significantly correlated with NSCLC patient survival (Fig. 1E, Fig. S1, $n=500$, high expression cases $=250$, low expression cases $=250, p=0.001$ ). Consistent with TCGA data analysis results (Fig. 1F), GEO data analysis also showed that NCAPG were highly expressed in NSCLC tumor tissues (Fig. 1G, $p<0.001$ ). Furthermore, NCAPG expression was significantly correlated with TNM stage $(n=970, p<0.001)$, T stage $(n=970, p<0.001)$, and $N$ stage $(n=970, p<0.001)$ in NSCLC patients (Table 1 ). The above results suggest that NCAPG expression is positively related to NSCLC.

To further investigate the expression and prognostic value of NCAPG, IHC was performed to assess NCAPG expression in NSCLC tumor tissues (Fig. 2A). Our study demonstrated that the expression level of NCAPG was correlated with gender, age, and histopathologic type (Table 2). KaplanMeier survival analysis showed that NCAPG expression was negatively correlated with survival in NSCLC patients (Fig. $2 \mathrm{~B}, n=156, p=0.003$ ), particularly in elderly patients ( $\geq 60$ years old) (Fig. $2 \mathrm{C}, n=84, p=0.0022$ ), adenocarcinoma (Fig. 2D, $n=92, p=0.0239$ ), and squamous cell carcinoma (Fig. 2E, $n=43, p=0.0296$ ). Cox regression analysis further demonstrated that NCAPG expression was an independent risk factor for NSCLC patient survival (Table 3, $p=0.022$ ). Importantly, both RT-qPCR and Western blot analyses showed that NCAPG expression was significantly increased in tumor tissues when compared with adjacent normal tissues (Fig. 2F, G). In addition, NCAPG was notably 
Table 3 Summary data for Cox proportional hazards regression analysis of the effect of clinicopathological characteristics and NCAPG expression on overall survival

\begin{tabular}{|c|c|c|c|c|}
\hline \multirow[t]{2}{*}{ subtype } & \multicolumn{2}{|l|}{ Univariate analysis } & \multicolumn{2}{|l|}{ Multivariate analysis } \\
\hline & Hazard ratio $(95 \% \mathrm{Cl})$ & $P$-value & Hazard ratio $(95 \% \mathrm{Cl})$ & $P$-value \\
\hline \multicolumn{5}{|l|}{ Gender } \\
\hline Female & 1 & & 1 & \\
\hline Male & $2.048(1.167-3.594)$ & 0.012 & $1.587(0.888-2.836)$ & 0.119 \\
\hline \multicolumn{5}{|l|}{ Age } \\
\hline$<60$ & 1 & & & \\
\hline$\geq 60$ & $1.150(0.679-1.947)$ & 0.603 & & \\
\hline \multicolumn{5}{|l|}{ TNM Stage } \\
\hline | / | & 1 & & 1 & \\
\hline III / IV & $3.033(1.784-5.155)$ & $<0.0001$ & $2.770(1.620-4.738)$ & $<0.0001$ \\
\hline \multicolumn{5}{|l|}{ Tumor stage } \\
\hline pT1 & 1 & & & \\
\hline pT2 & $2.145(0.889-5.172)$ & 0.089 & & \\
\hline pT3 & $3.436(1.301-9.077)$ & 0.013 & & \\
\hline \multicolumn{5}{|l|}{ Node metastasis } \\
\hline pNO & 1 & & & \\
\hline $\mathrm{pN} 1 / \mathrm{pN} 2$ & $1.114(0.579-2.141)$ & 0.747 & & \\
\hline \multicolumn{5}{|l|}{ Histological type } \\
\hline Adenocarcinoma & 1 & & & \\
\hline Squamous cell carcinoma Differentiated degree & $1.424(0.772-2.625)$ & 0.258 & & \\
\hline Poorly differentiated & 1 & & & \\
\hline Moderately differentiated & $1.093(0.539-2.215)$ & 0.806 & & \\
\hline Highly differentiated NCAPG expression & $0.816(0.255-2.610)$ & 0.732 & & \\
\hline Negative & 1 & & 1 & \\
\hline Positive & $2.353(1.296-4.272)$ & 0.005 & $2.022(1.1 .06-3.696)$ & 0.022 \\
\hline
\end{tabular}

Cl confidence interval

overexpressed in six NSCLC cell lines, with the highest expression in A549 cells (Fig. 2H, I).

\section{Depletion of NCAPG inhibits proliferation, migration, and invasion of NSCLC cells}

A549 cells transfected with either a control shRNA or shRNAs specifically against NCAPG were detected by Western blot, and the NCAPG inhibitor shRNA3 was the most effective inhibitor of NCAPG and was chosen to treat NSCLC cells (Fig. 3A). Both mRNA and protein expression levels of NCAPG were significantly decreased following NCAPG knockdown in A549 (Fig. 3B, $p$ $<0.001$ ) and H1299 cells (Fig. 2S E, F, $p<0.05$ ). In addition, NCAPG knockdown significantly inhibited cell proliferation, migration, and invasion (Fig. 3C-E, $p<0.001$; Fig. 2S G-I, $p<0.0001)$. Furthermore, we also have used shRNA2 to transfect NSCLC cells, and achieved similar results that NCAPG knockdown inhibited cell proliferation, migration, and invasion (data not shown).

To explore whether NCAPG could influence NSCLC tumor growth in vivo, we performed xenograft experiments on BALB/c nude mice by subcutaneous injection of $\mathrm{H} 1299$ cells transfected with either LV-shNCAPG or LV-shCtrl. Immunohistochemistry of xenograft tumor tissues showed that Ki-67 expression was reduced in the LV-shNCAPG tumors (Fig. 3F). These results showed that $N C A P G$ knockdown significantly suppressed tumor growth, and that volume and weight of the LV-shNCAPG xenografts were notably decreased compared with that of the LV-shCtrl tumors (Fig. 3F-H, $p<0.01)$. These data were independently confirmed using A549 xenografts transfected with LV-shNCAPG plasmid (Fig. S2 A-D, $p<0.05$ ).

In addition, SPARC, a protein regulating the epithelial-mesenchymal-paracrine signaling [17], was also significantly suppressed (Fig. 3F). We suspect that NCAPG might play a role in tumor metastasis. To assess the relationship between NCAPG and NSCLC tumor metastasis in vivo, we established a pulmonary metastasis tumor model in nonobese diabetic/severe (NOD/SCID) mice, and observed that silencing NCAPG in H1299 cells suppressed lung metastases (Fig. 3I, J), indicating that the 


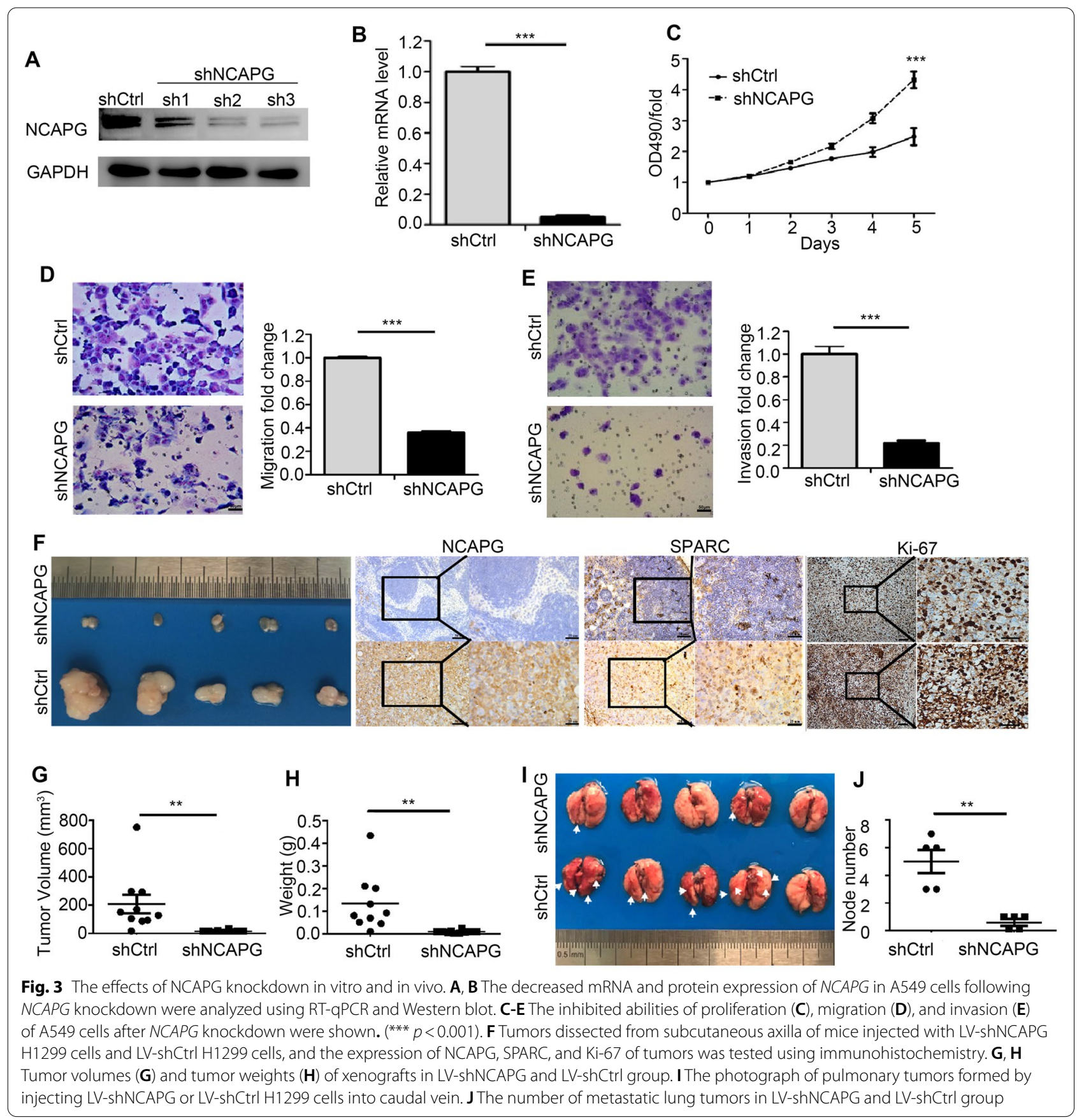

metastasis ability of tumor is significantly inhibited following NCAPG knockdown. However, whether NCAPG promotes NSCLC tumor metastasis through SPARC should be further studied.

\section{Ncapg deficiency suppresses urethane-induced lung cancer in vivo}

To further investigate the role of Ncapg in urethaneinduced lung cancer, urethane was administered to
Ncapg-deficient mice. We generated Ncapg gene knockout constructs and crossed heterozygous targeted mice to generate $\mathrm{Ncapg}^{-1-}$ mice. Due to the embryonic lethality after Ncapg knockout (Fig. S3 A, B), only $\mathrm{Ncapg}^{+/-}$and $\mathrm{Ncapg}^{+/+}$mice could be detected using PCR and agarose gel electrophoresis to test mouse embryonic fibroblasts (MEFs) at embryonic day 9 (Fig. S3 C, D). It has established that consistently repeated urethane exposure results in pulmonary adenoma and 


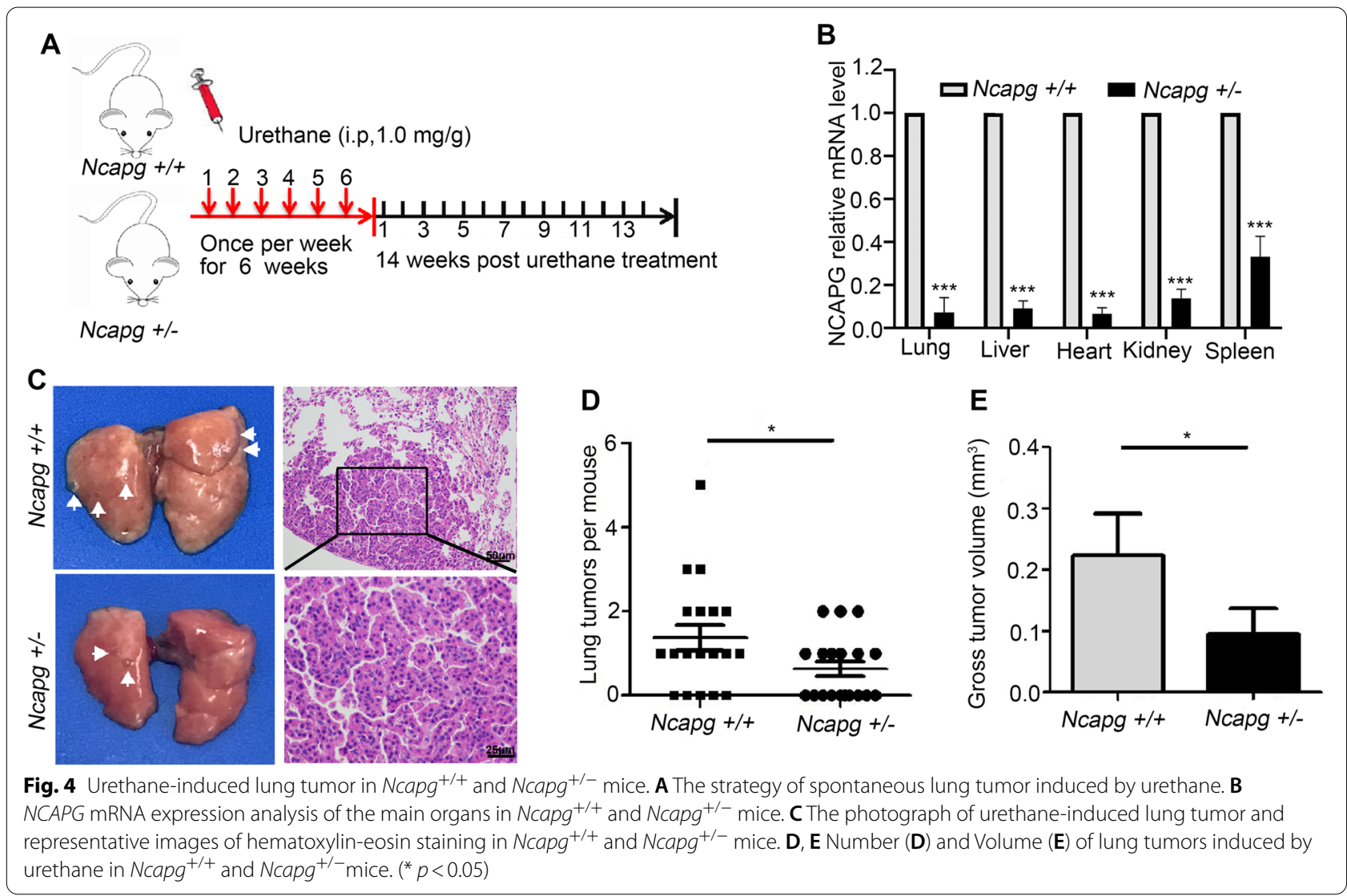

adenocarcinoma formation [18]. Therefore, Ncapg ${ }^{+/-}$ and wide type C57BL/6 mice were all injected intraperitoneally with urethane to induce lung tumor (Fig. 4A). We confirmed that the mRNA expression of NCAPG in organs of $\mathrm{Ncapg}^{+/-}$mouse was attenuated (Fig. 4B). The results indicated that the number of tumor nodules formed in $\mathrm{Ncapg}^{+/-}$mouse lung tissues was significantly less than that of wide type group (Fig. 4C-E). These data indicate that NCAPG plays a significant role in lung tumorigenesis.

\section{LGALS1 interacts with NCAPG to mediate tumor progression in NSCLC cells}

To elucidate the mechanism by which NCAPG promotes cell proliferation, migration, and invasion in
NSCLC, we precipitated and analyzed NCAPG-interacting proteins in A549 cells using Co-Immunoprecipitation (Co-IP) and Liquid Chromatography-Mass Spectrometry (LC-MS), respectively (Fig. 5A). NCAPGinteracting protein networks were scored based on their correlation with DNA replication, recombination, repair, and metabolism functions. LGALS1 (Galectin-1), a $\beta$-galactoside binding mammalian lectin, is overexpressed in NSCLC and is associated with cancer progression and immune disorders. We used INGENUITY ${ }^{\circledR}$ database analysis and functional enrichment analysis to identify that LGALS1 was co-precipitated with NCAPG in A549 cells (Fig. 5B, C). Furthermore, we detected a clear positive signal showing the interaction of NCAPG and LGALS1 in A549 cells by PLA coupled

(See figure on next page.)

Fig. 5 LGALS1 was identified as an important NCAPG-interacting protein. A The SDS-PAGE image with Coomassie Blue Staining before mass spectrometry identified NCAPG-interacting proteins in A549 cells. B The highest score of NCAPG-interacting protein network was measured with biological function enrichment analysis. C LGALS1 was co-precipitated by NCAPG in A549 cells. D For each panel, the images from left to right showed PLA signal in A549 cells (red), cell nuclei stained by DAPI (blue), and overlays of the two images. Scale bar $20 \mu \mathrm{m}$. E The histogram displays the relative fluorescent value of PLA punctuates and nuclei. F PLA signal of A549 cells for different conditions by flow cytometer. G Western blot analysis for LGALS1 protein after LGALS1 knockdown in A549 cells. H-M The abilities of proliferation, migration, and invasion were decreased after LGALS1-knockdown in H1299 and A549 cells. Scale bar $50 \mu m$. .** $\left.p<0.01,{ }^{* * *} p<0.001\right)$ 


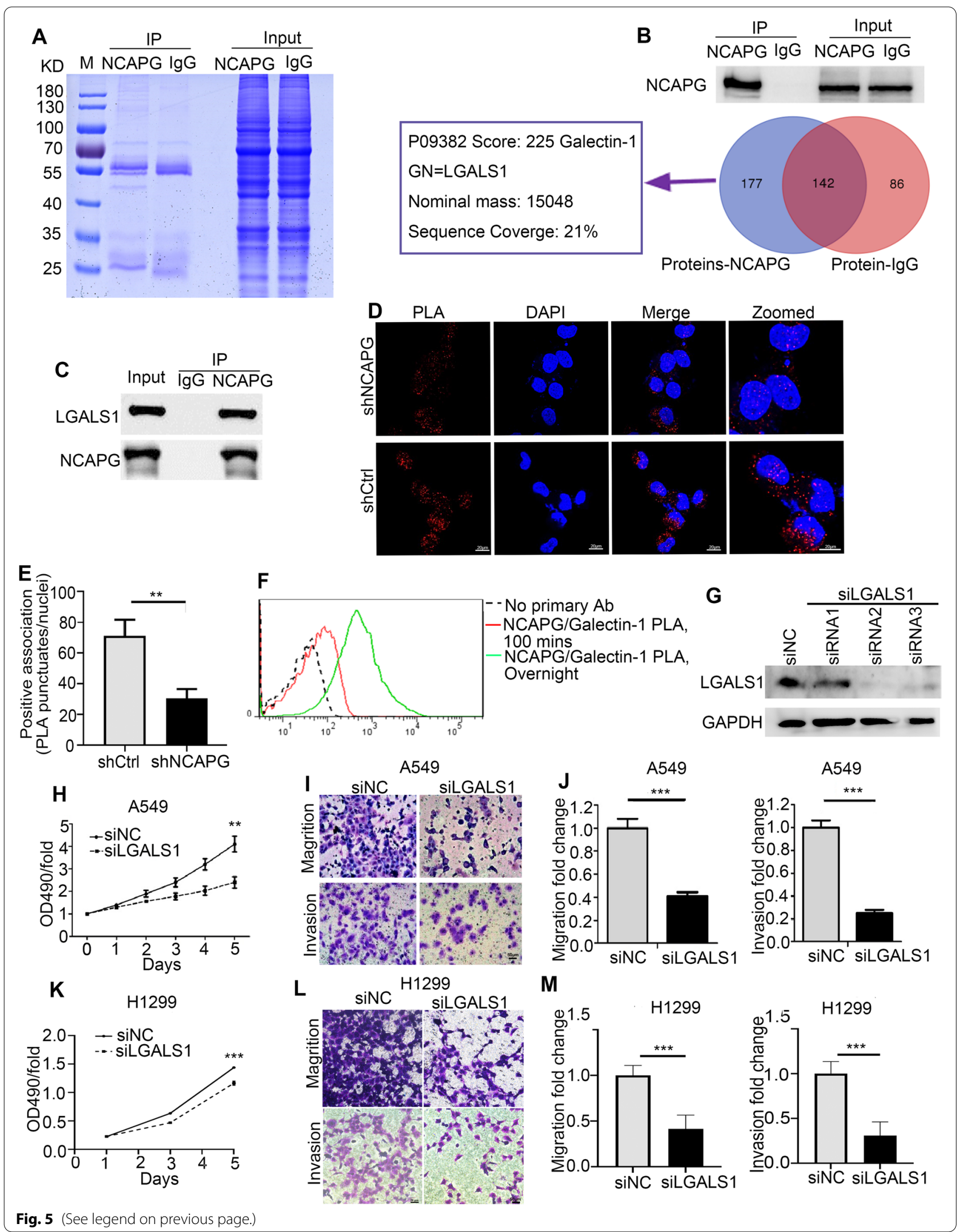




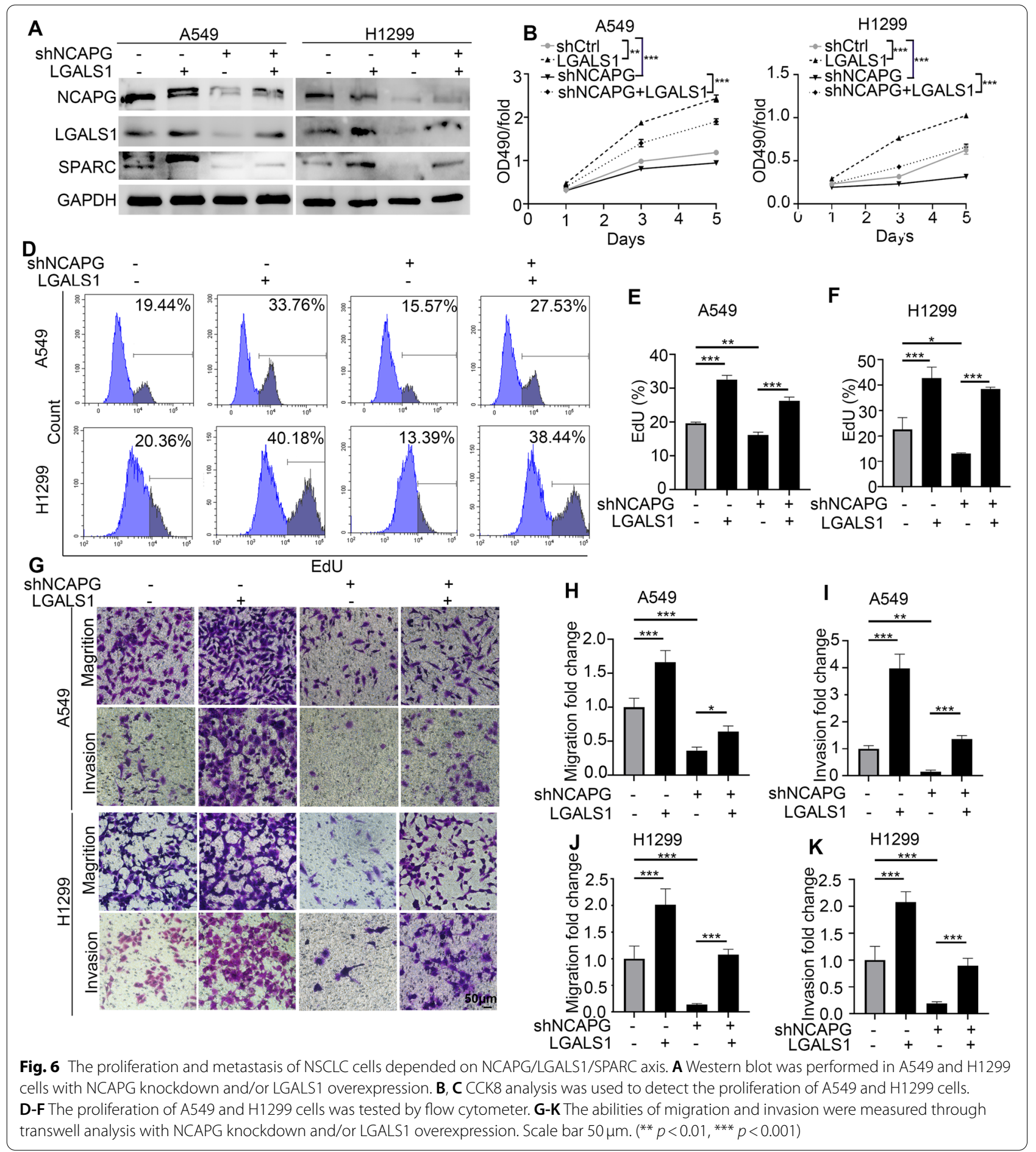

with immunofluorescence assays (Fig. 5D, E) and flow cytometer (Fig. 5F). Similarly, the effect of LGALS1 knockdown also inhibited cell proliferation, migration, and invasion in A549 and H1299 cells (Fig. 5G-M).

To confirm whether NCAPG induced NSCLC cells proliferation and metastasis through LGALS1, Western blot were performed to show that NCAPG inhibition remarkably suppressed LGALS1 and SPARC protein levels, while downregulation of SPARC was reversed in LGALS1 overexpression cells (Fig. 6A). MTT, flow cytometry, and transwell analysis were used to detect the proliferation, migration, and invasion of H1299 and A549 cells with 
NCAPG reduction and/or LGALS1 forced overexpression (Fig. 6B-K). These data showed the functions of proliferation, migration, and invasion in NSCLC cells might partially depend on NCAPG/LGALS1/SPARC axis.

\section{Discussion}

NCAPG has been demonstrated to regulate DNA location on chromosomes during mitotic chromosome condensation regulated by the condensin I complex in mitosis process, as a potential candidate target for medicine development. This study discovered that NCAPG expression was negatively associated with NSCLC survival and played a major role in the initiation and progression of NSCLC in vitro and in vivo. Our study suggests the important role of NCAPG in NSCLC and its potential value in diagnosis and prognosis, suggesting that NCAPG represent a promising target for NSCLC treatment. It is critical to identify novel biomarkers and targets for improving NSCLC patients' clinical outcomes. Using comprehensive analyses of NSCLC cases from the TCGA and the GEO databases, we found that NCAPG was significantly overexpressed in NSCLC tumor tissues and negatively correlated with overall patient survival. Consistent with our findings, previous studies suggest that NCAPG functions as oncogene in cancer cells [9], whose overexpression could promote epithelial-mesenchymal transition, proliferation, and apoptosis suppression in tumor cells $[19,20]$. We next investigated the underlying mechanism of NCAPG-mediated proliferation and metastasis in NSCLC cells. Our data revealed that NCAPG interacts with LGALS1 protein resulting in increased cell proliferation, invasion, and migration in vitro and in vivo. The galectin family members of glycan-binding proteins are known for their role in regulating cancer development and progression [21]. LGALS1, a glycan-binding protein, regulates tumor cell proliferation, invasion, and metastasis in pancreatic ductal adenocarcinoma [22]. As a result of our findings, we suggest that NCAPG interacts with LGALS1 to promote proliferation, invasion, and migration in NSCLC cells. Since SPARC overexpression enhances tumor-initiated permeability and vascular leakiness, which finally induces lung metastasis [17], and our study found that NCAPG knockdown could suppress SPARC expression. We speculate that NCAPG might interact with LGALS1 to upregulate SPARC, leading to activate EMT signaling and augment tumor-derived vascular permeability.

With the rapid development of NSCLC treatments over the last two decades, a widespread use has been limited by the fact that targeted therapies require specific genetic background and resistance often occurs. Hence, development of new therapeutic targets is needed. Urethane can combine with DNA to form DNA etheno adducts and is used to artificially develop pulmonary adenomas in neonatal mice [23]. We found that NCAPG played a significant role in lung tumorigenesis of a urethane-induced murine lung cancer model. Since NCAPG has been proposed as therapeutic target to overcome trastuzumab resistance in breast cancer [10], our results indicate that NCAPG is not only an important oncogenic driver but may also serve as a new target for treating NSCLC. Future work is warranted to elucidate the mechanisms by which NCAPG knockout induces embryonic lethality.

In conclusion, $N C A P G$ is required for tumorigenesis and progression of NSCLC. NCAPG represents a promising biomarker for NSCLC early diagnosis, prognosis prediction, and drug development. Inhibition of the NCAPG/ LGALS1 axis may be a new strategy for NSCLC treatment.

\begin{abstract}
Abbreviations
ACN: Acrylonitrile Acn; ALK: Anaplastic Lymphoma Kinase; ATCC: American Type Culture Collection; BRAF: B-Rapidly Accelerated Fibrosarcoma; Cl: Confidence Interval; Co-IP: Co-Immunoprecipitation; EDTA: Ethylene Diamine Tetraacetic Acid; EGFR: Epidermal Growth Factor Receptor; DMEM: Dulbecco's Modified Eagle Medium; FBS: Fetal Bovine Serum; FC: Fold Change; FDR: False Discovery Rate; GO: Gene Ontology; HRP: Horseradish Peroxidase; IHC: Immunohistochemistry; KEGG: Kyoto Encyclopedia of Genes and Genomes; KM: KaplanMeier; KRAS: Kirsten Rat Sarcoma Viral Oncogene; LC-MS: Liquid Chromatography-Mass Spectrometry; MEFs: Mouse Embryonic Fibroblasts; MET: MNNG HOS Transforming Gene; NCAPG: Non-SMC Condensin I Complex Subunit G; NOD/SCID: Non-Obese Diabetes/Server Combined Immune-Deficiency; NSCLC: Non-Small Cell Lung Cancer; ROS: Reactive Oxygen Species; RPMI: Roswell Park Memorial Institute Medium; RT-qPCR: Realtime Quantitative PCR; SDS-PAGE: Sodium Dodecyl Sulfate Polyacrylamide Gel Electrophoresis; siRNA: Small Interfering RNA; shRNA: Short Hairpin Interfering RNA; TCGA: The Cancer Genome Atlas; TFA: Trifluoroacetic Acid; TNM: Tumor-node-metastasis; WB: Western Blot.
\end{abstract}

\section{Supplementary Information}

The online version contains supplementary material available at https://doi. org/10.1186/s12943-022-01533-9.

Additional file 1.

\section{Acknowledgements}

Not applicable.

\section{Authors' contributions}

Haiqing Ma and Christophe Nicot contributed to the overall study design. Huanhuan Sun, Hong Zhang, Yushi Li, Yan Yan, and Cuiling Zhou performed experiments in vitro and in vivo. Gang Che performed the statistical analyses. Huanhuan Sun, Hong Zhang, Haiqing Ma, and Christophe Nicot wrote and revised the paper. All authors contributed to data interpretation and commented on the manuscript. The author(s) read and approved the final manuscript.

\section{Funding}

This research was supported by the National Natural Science Foundation of China $(81872308,82072719)$ and the Natural Science Foundation of the Guangdong Province (2021A15150100790).

Availability of data and materials

Not applicable. 


\section{Declarations}

\section{Ethics approval and consent to participate}

In this study, ethics approval was provided by Guangdong Provincial People's Hospital and the Fifth Affiliated Hospital of Sun Yat-sen University (KY-Z2021-299-01, KY-Z-2021-061, L030-1). This study was performed following the ethical standards of the Declaration of Helsinki.

\section{Consent for publication}

Not applicable.

\section{Competing interests}

All authors consented to publication in its present form.

\section{Author details}

'Medical Research Center, Guangdong Provincial People's Hospital, Guangdong Academy of Medical Sciences, 106 Zhongshan Er Rd, Guangzhou 510080, Guangdong, China. ${ }^{2}$ Department of Oncology, The Fifth Affiliated Hospital, Sun Yat-sen University, Zhuhai, China. ${ }^{3}$ Department of Oncology, Guangdong Cardiovascular Institute, Guangdong Provincial People's Hospital, Guangdong Academy of Medical Sciences, Guangzhou, China. ${ }^{4}$ Department of Pathology and Laboratory Medicine, University of Kansas Medical Center, 3901 Rainbow Boulevard, Kansas City, KS 66160, USA.

Received: 3 November 2021 Accepted: 8 February 2022

Published online: 18 February 2022

\section{References}

1. Remon J, Steuer CE, Ramalingam SS, Felip E. Osimertinib and other third-generation EGFRTKI in EGFR-mutant NSCLC patients. Ann Oncol. 2018;29(suppl_1):i20-i7.

2. Shaw AT, Solomon BJ, Besse B, Bauer TM, Lin CC, Soo RA, et al. ALK resistance mutations and efficacy of Lorlatinib in advanced anaplastic lymphoma kinase-positive non-small-cell lung Cancer. J Clin Oncol. 2019;37(16):1370-9.

3. Zhuo M, Liang Z, Yi Y, Wu N, Yang X, Zhong J, et al. Analysis of MET kinase domain rearrangement in NSCLC. Lung Cancer. 2020;145:140-3.

4. Ferrer I, Zugazagoitia J, Herbertz S, John W, Paz-Ares L, Schmid-Bindert G. KRAS-mutant non-small cell lung cancer: from biology to therapy. Lung Cancer. 2018:124:53-64.

5. Planchard D, Smit EF, Groen HJM, Mazieres J, Besse B, Helland A, et al. Dabrafenib plus trametinib in patients with previously untreated BRAF(V600E)-mutant metastatic non-small-cell lung cancer: an openlabel, phase 2 trial. Lancet Oncol. 2017:18(10):1307-16.

6. Drilon A, Siena S, Dziadziuszko R, Barlesi F, Krebs MG, Shaw AT, et al. Entrectinib in ROS1 fusion-positive non-small-cell lung cancer: integrated analysis of three phase 1-2 trials. Lancet Oncol. 2020;21(2):261-70.

7. Eberlein A, Takasuga A, Setoguchi K, Pfuhl R, Flisikowski K, Fries R, et al. Dissection of genetic factors modulating fetal growth in cattle indicates a substantial role of the non-SMC condensin I complex, subunit G (NCAPG) gene. Genetics. 2009;183(3):951-64.

8. Kimura K, Cuvier O, Hirano T. Chromosome condensation by a human condensin complex in Xenopus egg extracts. J Biol Chem. 2001;276(8):5417-20.

9. Sun DP, Lin CC, Hung ST, Kuang YY, Hseu YC, Fang CL, et al. Aberrant expression of NCAPG is associated with prognosis and progression of gastric Cancer. Cancer Manag Res. 2020;12:7837-46.

10. Jiang L, Ren L, Chen H, Pan J, Zhang Z, Kuang X, et al. NCAPG confers trastuzumab resistance via activating SRC/STAT3 signaling pathway in HER2-positive breast cancer. Cell Death Dis. 2020;11(7):547.

11. Liu W, Liang B, Liu H, Huang Y, Yin X, Zhou F, et al. Overexpression of nonSMC condensin I complex subunit G serves as a promising prognostic marker and therapeutic target for hepatocellular carcinoma. Int J Mol Med. 2017:40(3):731-8.

12. Liu ZK, Zhang RY, Yong YL, Zhang ZY, Li C, Chen ZN, et al. Identification of crucial genes based on expression profiles of hepatocellular carcinomas by bioinformatics analysis. PeerJ. 2019;7:e7436.
13. Wei W, Lv Y, Gan Z, Zhang Y, Han X, Xu Z. Identification of key genes involved in the metastasis of clear cell renal cell carcinoma. Oncol Lett. 2019:17(5):4321-8.

14. Wang H, Yao H, Li C, Shi H, Lan J, Li Z, et al. HIP1R targets PD-L1 to lysosomal degradation to alter T cell-mediated cytotoxicity. Nat Chem Biol. 2019;15(1):42-50

15. Mackey-Cushman SL, Gao J, Holmes DA, Nunoya Jl, Wang R, Unutmaz D, et al. FoxP3 interacts with linker histone H1.5 to modulate gene expression and program Treg cell activity. Genes Immun. 2011;12(7):559-67.

16. Ma H, Liang X, Chen Y, Pan K, Sun J, Wang H, et al. Decreased expression of BATF2 is associated with a poor prognosis in hepatocellular carcinoma. Int J Cancer. 2011;128(4):771-7.

17. Tichet M, Prod'Homme V, Fenouille N, Ambrosetti D, Mallavialle A, Cerezo $M$, et al. Tumour-derived SPARC drives vascular permeability and extravasation through endothelial VCAM1 signalling to promote metastasis. Nat Commun. 2015;6:6993.

18. Shimkin MB, Polissar MJ. Some quantitative observations on the induction and growth of primary pulmonary tumors in strain a mice receiving urethan. J Natl Cancer Inst. 1955;16(1):75-97.

19. Gong C, Ai J, Fan Y, Gao J, Liu W, Feng Q, et al. NCAPG promotes the proliferation of hepatocellular carcinoma through PI3K/AKT signaling. Onco Targets Ther. 2019;12:8537-52.

20. Zhang X, Zhu M, Wang H, Song Z, Zhan D, Cao W, et al. Overexpression of NCAPG inhibits cardia adenocarcinoma apoptosis and promotes epithelial-mesenchymal transition through the Wnt/beta-catenin signaling pathway. Gene. 2021;766:145163.

21. Wu H, Song S, Yan A, Guo X, Chang L, Xu L, et al. RACK1 promotes the invasive activities and lymph node metastasis of cervical cancer via galectin-1. Cancer Lett. 2020;469:287-300

22. Orozco CA, Martinez-Bosch N, Guerrero PE, Vinaixa J, Dalotto-Moreno T, I glesias M, et al. Targeting galectin-1 inhibits pancreatic cancer progression by modulating tumor-stroma crosstalk. Proc Natl Acad Sci U S A. 2018;115(16):E3769-E78.

23. Barbin A. Etheno-adduct-forming chemicals: from mutagenicity testing to tumor mutation spectra. Mutat Res. 2000;462(2-3):55-69.

\section{Publisher's Note}

Springer Nature remains neutral with regard to jurisdictional claims in published maps and institutional affiliations.

Ready to submit your research? Choose BMC and benefit from

- fast, convenient online submission

- thorough peer review by experienced researchers in your field

- rapid publication on acceptance

- support for research data, including large and complex data types

- gold Open Access which fosters wider collaboration and increased citations

- maximum visibility for your research: over 100M website views per year

At BMC, research is always in progress.

Learn more biomedcentral.com/submissions 